\title{
Effects of Moderate-Intensity Intermittent Circuit Training in Obese and Overweight Individuals
}

\author{
Irene-Chrysovalanto Themistocleous ${ }^{1}$, Petros Agathangelou ${ }^{2}$ and Manos Stefanakis ${ }^{1^{*}}$
}

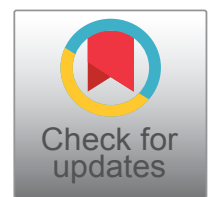

\author{
${ }^{1}$ Department of Health and Life Sciences, University of Nicosia, Cyprus \\ ${ }^{2}$ Department of Medicine, University of Nicosia, Cyprus
}

*Corresponding author: Manos Stefanakis, Department of Life and Health Sciences, Department of Physiotherapy, University of Nicosia, 8, Markou Drakou Str., Egkomi, Research and Technology Building, CY-2409, Cyprus, Fax: $+35722842555$

\begin{abstract}
Background: Obesity has become a serious problem in Western societies and is associated with several health problems and reduced quality of life. The purpose of this randomised, clinical trial is to investigate the effects of a moderate-intensity, intermittent, circuit training program on a range of cardiovascular risk factors such as body composition, biochemical and physiological variables and aerobic capacity in obese and overweight individuals.

Methods: A total of 30 participants were selected and 15 of them performed a moderate-intensity, intermittent, circuit training program; whereas the rest acted as a control-group. The circuit training program consisted of 10 exercise stations and was performed 2 times per week for 8-weeks in total. The experimental group performed a circuit training program with training cycles at $50-60 \%$ of heart rate reserve which were followed by active recovery periods at $40-50 \%$ of heart rate reserve. Control-group remained sedentary during the period of 8-weeks and follow-up. Biochemical markers, isometric strength, body composition and physiological parameters were assessed at baseline and 8-weeks post-intervention, whereas maximal oxygen consumption was assessed at baseline, 8-weeks and 2-months follow-up.

Results: Significant improvements were demonstrated in BW (2.5\%), BMI ( 3\%), BF\% ( 4\%), BF-Kg ( 6\%), and LBM $\%(3.3 \%)$, SBP (5.3\%), HR-resting (4.3\%), MAP ( 5\%) and cardiorespiratory fitness $(\sim 11 \%)$ in the exercise group post-intervention. Significant time by group interaction was found for all physiological variables (SBP, DBP, HR, MAP, RPP) in favour of the exercise group. VO2peak improved post-intervention by $\sim 11 \%$ in the CT-group, in contrast to $1.7 \%$ in the control-group.
\end{abstract}

Conclusion: The present data suggest that a moderate-intensity, intermittent circuit training program for 8-weeks may be effective in reducing cardiovascular risk factors.

\section{Keywords}

Circuit training, Body composition, Cardiovascular health, Risk factors, Obese, Overweight, Moderate-intensity intermittent training

\section{Introduction}

Obesity is described as weight gain along with body fat accumulation that is associated with an increased risk of certain chronic diseases [1]. Epidemiological data suggest that obesity increases globally [2] and is strongly associated with the development of chronic diseases, lack of health-related quality of life and rising costs of health care [3]. In order to lose weight, someone needs to sustain a negative energy equilibrium and the most effective way to achieve this is a combination of diet, exercise, and behavioural change [4,5]. Specifically, an increased energy expenditure through exercise combined with a proper diet can lead to weight loss that lasts up to 36-months [6]. In addition, exercise is important in improving cardiorespiratory fitness, which is independently associated with overall mortality [7].

However, despite the body of evidence on the benefits of regular exercise, as well as the negative effects of a sedentary lifestyle, most obese individuals

Citation: Themistocleous IC, Agathangelou P, Stefanakis M (2021) Effects of Moderate-Intensity Intermittent Circuit Training in Obese and Overweight Individuals. Int J Sports Exerc Med 7:194. doi. org/10.23937/2469-5718/1510194

Accepted: June 12, 2021; Published: June 14, 2021

Copyright: (C) 2021 Themistocleous IC, et al. This is an open-access article distributed under the terms of the Creative Commons Attribution License, which permits unrestricted use, distribution, and reproduction in any medium, provided the original author and source are credited. 
have very low levels of physical activity, well below the recommended physical activity per week (150 min of moderate or 75 min of vigorous activity) [8]. It seems that this recommendation is not realistic for most obese and overweight individuals due to lack of sufficient time to spend on prolonged exercise regimes [9]. In order to overcome the time barrier, High-Intensity Interval Training (HIIT) was suggested as a useful and time-consuming alternative option to ModerateIntensity Continuous Training (MICT) [10]. Whyte, et al. (2010) showed that 2-weeks of sprint interval training ( 6 sessions of 4-6 $\times 30$ s and 4-5 mins recovery) improved insulin sensitivity, Waist Circumference (WC) and Systolic Blood Pressure (SBP) in overweight and obese men [11]. Moreover, Trilk, et al. showed that overweight and obese women had $12 \%$ increase in VO2max, $11.4 \%$ increase in stroke volume and $8.1 \%$ reduction in Heart Rate (HR) at rest following 4-weeks of sprint interval training $(4-7 \times 30$ s "all out" sprints and 4 mins recovery) [12]. Even a single bout of HIIT ( $4 \times 30$ s "all out" sprints with 4-mins recovery) as Whyte, et al. showed, improved glucose tolerance, insulin sensitivity and fat oxidation in obese men [13]. Other studies conducted with overweight and obese individuals haveused a modified HIIT protocol for 2-6-weeks, which is likely to be more practical for overweight and obese populations than Wingate and reported improvements in body composition, insulin sensitivity, lipid profile and V02max [14-17].

Although the findings from these studies suggest that the "all out" and modified HIIT protocolscan be an effective way to manage obesity, injuries often become a problem among the sedentary people who are unfamiliar with exercise of such high intensity [18]. In addition such an exercise regime often requires specific equipment and guidance from exercise professionals through-out the session [18].

On the other hand, if exercise intensity is moderate, the dropout rate among sedentary or untrained individuals is usually lower and also the rate of adherence is higher than in vigorous exercise [19]. Collectively these results provide evidence that obese and overweight individuals are either not willing or unable to participate in a strenuous exercise like HIIT [20]. The moderate intensity makes the exercise significantly easier and more enjoyable to unfit and overweight individuals therefore has the potential to increase adherence.

To date, the majority of HIIT studies in overweight and obese individuals have used cycling and running protocols, with a weekly frequency of 3-times per week and a follow-up time /8-16-weeks [21-25]. This focus on just one exercise mode, is contrary to the public health guidelines which suggest that in addition to aerobic physical exercise, moderate to vigorous musclestrengthening $\geq 2$ days/week will also bring other health benefits [26]. Apart from the health benefits, strength adaptations might be vital for increasing enjoyment of exercise [27] as resistance exercise leads to an increase in muscle strength and power [28], which improves bone mineral density [29], lipid profile [30], glycaemic control [31], metabolic syndrome risk factors [32] and body composition [33]. Additionally, the improvements in strength are associated with enhancements in muscle performance and physical function, which translate infavourable changes in the activities of daily living in obese and overweight individuals [34].

It was therefore suggested that Circuit Training (CT), which combines aerobic and resistance training, may be the preferred way of producing complex adaptations than a unimodal exercise regimen such as HIIT [33,35]. During exercise the use of body weight as resistance or simple and cheap equipment, combined with short-periods of active rest has the potential to eliminate training time and be cost effective [36]. The proposed exercise program consists of a combination of resistance and aerobic exercises, using inexpensive and readily accessible equipment to overcome some of the current exercise problems that general population and especially overweight and obese individuals may have. CT effectively reduces the amount of time the individual spends exercising while helping to achieve a satisfactory mode of training [37]. Evidence suggests that CT can improve body composition [38-42], cardio metabolic risk factors $[43,44]$, biochemical markers [45], physiological parameters [46], $\mathrm{VO}_{2} \max [10]$ and more importantly, motivation to exercise [47]. Despite the after-mentioned improvements in health with CT it is still not clear what is the most effective combination of exercise parameters (frequency, intensity, type, time, work effort to rest ratio) in order to promote health of obese and overweight population. In addition, it is not clear what is the minimum volume of $\mathrm{CT}$ training above which meaningful adaptations appear.

The objective of the study was to examine the effects of a short duration (20 min/session), moderate intensity, intermittent, CT, with low-cost equipment, in apparently healthy obese and overweight individuals. It was hypothesized that a CT program performed twice per week for 8-weeks will improve at least some of the body composition, biochemical markers, lower-body strength, cardiorespiratory fitness and physiological parameters in obese and overweight population.

\section{Material and Methods}

\section{Participants}

The study was approved by the Cyprus Bioethics Committee (EЕВK/EП/2017/38). This randomized controlled clinical trial included 30 adults, between 1855 years of age, with body mass index $(\mathrm{BMI}) \geq 25 \mathrm{~kg} / \mathrm{m}^{2}$ which were free of musculoskeletal or cardiovascular conditions that would impede participation in an 
exercise program. The participants were recruited after an announcement in a noticeboard in the local area of Nicosia, Cyprus. Participants were excluded if they had regular physical activity over the past 6-months and any symptoms related to cardiopulmonary diseases. Prior to the study, participants were informed about the aim and the design of study before signing a written informed consent. After a general assessment by a cardiologist, those who met the inclusion criteria were randomized into two groups, CT-group ( $\mathrm{n}=$ 15 ) or control-group without exercise ( $n=15)$ using established randomization software (SealedEnvelope. com, Clerkenwell Workshops). Participants were required to follow their typical diet and activities of daily life throughout the study. It was not possible to blind participants, as they are usually aware whether they are training or not [48], but the biochemical analysis and the six minute walk test (6MWT) were performed by blind assessors.

\section{Experimental design}

Participants were assessed by two investigators at baseline (T-1), after an 8-week intervention (T-2) and 2-months after the intervention (T-3). Anthropometric, biochemical markers, strength of lower body and physiological parameters measurements were performed at $\mathrm{T}-1$ and $\mathrm{T}-2$, whereas $\mathrm{VO}_{2}$ peak and level of fitness were assessed at all 3-time points. Moreover, rating of exertion was assessed only in the first session (pre and post). Baseline assessments were performed under laboratory conditions 1-week before intervention, whereas the follow-up assessments were carried out at least 2-days apart from the last exercise session and after 2-months, at a similar time of the day. Measurements were taken between 8:00-9:00 a.m. after an over-night fast, with no consumption of alcohol or caffein and participation in vigorous physical activity at least 24-hours prior to assessment. On the first visit, all outcome measures were assessed except biochemical markers and cardiorespiratory fitness which were tested on the second visit (24-hours later).

\section{Measurements}

\section{Biochemical markers measurement}

Under 12-hours of fasting state, at T-1and T-2 (24-hoursafter the completion of the program), $5 \mathrm{ml}$ of blood was drawnby a trained biochemist from the antecubital vein in sitting position at 08:00 a.m. Blood markers including total cholesterol, triglycerides, HighDensity Lipoprotein cholesterol (HDL), Low-Density Lipoprotein cholesterol (LDL), and glucose were analysed with the Lx20 analyser (Beckman Coulter Inc., Fullerton, CA).

\section{Blood pressure and heart rate measurements}

Resting Systolic (SBP) and Diastolic Blood Pressure (DBP) and resting Heart Rate (HR) were measured at T-1,
T-2 and at each session (pre-training and post-training). Participants rested in a seated position for 10-min, before resting Blood Pressure (BP) was measured using an automatic BP monitor (Omron M3, Kyoto, Japan). The measurements were taken 3-times (1-min apart) in order to take the average. The resting heart rate (HRrest) was measured also in a seated position after $10-\mathrm{min}$ of rest by the pulse meter watch (Polar V800, Finland). To set the intensity of training, maximum HR (HRmax) was predicted fromthe 208 - $(0.7 \times$ age) formula even if participants were older than 40 years [49] and then the intensity of the exercise was set as a percentage of the heart rate reserve (\%HRR) for each participant. HRR was determined by theformula: Target HR (THR) $=[(\mathrm{HRmax})$ peak - HRrest) $\times \%$ intensity desired] + HRrest [50].

\section{Perceived exertion measurement}

Rating of exertion was recorded as a way to monitor exercise load using OMNI-RES ( 0 = extremely easy to 10 = extremely hard) [51]. Instructions were given to the participants before each session. Moreover, participants were instructedto rate their overall perception about how hard the exercise felt, at the end of each of the two cycles of the exercise.

\section{Anthropometric and body composition measure- ments}

The measurements conducted at the same time of day and evaluated with the same minimal clothes, without socks and shoes. Body composition measurements included lean body mass (LBM-Kg), percent Lean Body Mass (LBM\%), fat mass (BM-Kg), percent Body Fat (\%BF), Body Mass Index (BMI). Body composition measurements were conducted using an electronic scale that was calibrated every time (Omron BF511, Kyoto, Japan). Standing height was measured using a Seca 202 stadiometer (Seca, Hamburg, Germany). The WC was measured midway between the lowest rib and the iliac crest and the hip circumference at the level of the symphysis with a tape. Subsequently the Waist-toHip Ratio (WHR) was calculated based on the equation WHR = waist circumference/hip circumference [50]. The aforementioned measurements were used to comparechanges in body composition from T-1 to T-2.

\section{6-minute walk test}

6MWT was performed at all 3-time points to evaluate the cardiorespiratory fitness. Participants walked as far as possible in a 30-m indoor corridor, with marks every $3 \mathrm{~m}$ across its entire length. The turnround point was marked with a cone and the starting line was marked on the floor using a tape in order to acknowledge the beginning and end of each lap. The $\mathrm{VO}_{2}$ peak was calculated using the American College of Sports Medicine $($ ACSM $)$ formula VO2peak $=\left(0.02^{*}\right.$ distance $\left.[\mathrm{m}]\right)$ - $\left(0.191^{*}\right.$ age $\left.[\mathrm{yr}]\right)-\left(0.07^{*}\right.$ weight $\left.[\mathrm{kg}]\right)+\left(0.09^{*}\right.$ height $[\mathrm{cm}])+\left(0.26^{*}\right.$ rate pressure product $\left.\left[{ }^{*} 10-3\right]\right)+2.45$ based 
on the total meters the participants have walked [50].

\section{Isometric strength}

The maximal isometric strength of quadriceps and hamstrings of both limbs was measured on MicroFET 2 dynamometer (Hoggan Health Industries Inc. West Draper, UT, USA) at T-1 and T-2. Measurements were taken in a standardised seated position with hips and knees fixed at $90^{\circ}$ of flexion with a goniometer. Participants sat on the treatment bed, with their back supported in a fully upright position andboth hands on the side. A stabilizing strap was placed over both thighs just distal to the hipjoint and another strap was used to stabilize the dynamometer above the ankle. Participantswere asked to gradually increase their muscle force to a maximum effort which would need tobe sustained for 3s. The «break technique» was applied by the examiner, which overpowers patient's maximum effort and therefore produces an eccentric muscle force [52]. The average muscle force from 3-repetitions was calculated to minimize measurement variability.

\section{Physiological Parameters}

Resting mean Arterial Pressure (MAP) and Rate Pressure Product (RPP) were calculated at T-1 and T-2, based on the equations RPP $=\mathrm{HR} X \mathrm{SBP}$ and MAP $=[(\mathrm{DBP}$ $X 2)+S B P] / 3$ ] as suggested by several publications [5355].

\section{Training program}

Prior to the beginning of the exercise program, participants were involved in a single session in order to familiarize with the exercises performed in the study. During familiarization session an emphasis was placed on learning the proper exercise techniques and the utilisation of OMNI scale. Participants were instructed to abstain from food, caffeine or alcohol at least 5-hours before each exercise session for the duration of 8-weeks. CT program was performed twice a week (with at least 1 day rest in between) under the supervision of a physiotherapist. The exercise protocol consisted of a 3-min warm-up (toe taps, heel taps, marching on the spot, step jack, split squat jack, skier jack) and a 3-min cool-down (toe taps, heel taps, quadriceps stretching, hamstrings stretching, triceps stretching, pectoralis stretching) at 40-50\% HRR. After warm-up, participants had to complete 2 circuits in each session, with 8 stations per circuit and 12 repetitions per station. Specifically, the program consisted of 6 cardiovascular (CV) stations and 2 Active Recovery (AR) stations, lasting 1-min and 30s respectively. So, there was $1 \mathrm{AR}$ station for every $3 \mathrm{CV}$ stations. The training intensity of each CV station and AR station was performed at 50-60\% HRR and $40-50 \%$ HRR respectively. The exercise session was performed as: (1) Medicine ball squat overhead throw, (2) High knees, (3) Squat jumps, (4) Wall push-ups or standing bicep curl, (5) Marching on the spot, (6) Bend over double triceps kickback, (7) Split squat medicine ball slams, (8) Two-handed dumbbell lateral step-up or squats. If the participant failed to reach at least 12 repetitions ona given set, the weight was reduced in the following set, whereas when $>12$ repetitions were reached the weight was increased in the following set (following the OMNI-RES scale 6 "somewhat hard").

\section{Monitoring of physical activity}

All participants had low levels of physical activity as measured by the International Physical Activity Questionnaire (IPAQ) except one in the control group and 2 in the CT group which had moderate physical activity levels. Participants were instructed to maintain their lifestyle (refraining from any additional physical activity) and dietary habits, throughout the study. The level of physical activity (outside the exercise program in the CT group) was confirmed with the IPAQ after the study for all participants.

\section{Statistical analyses and sample size calculation}

Analyses were performed using SPSS vs. 25.0 (SPSS Inc., Chicago, IL). Equality of variance was assessed using Levene's test and the normality of the data was assessed using the Shapiro-Wilk statistic. Repeated measures ANOVA ( 2 groups $\times$ 2-time points) was applied for all assessment measures (or Friedman's test for non-normally distributed data), except for VO2peak and level of fitness which had 3-time points. Baseline characteristics (CT-group vs. Control-group) were compared with independent samples t-tests. The level of significance was set at $p<0.05$. Data are presented as mean \pm SD for normally-distributed data and median (interquartile range) for non-normally distributed data. Effect sizes for the Group $\times$ Time interaction were calculated using eta-squared $\left(\eta^{2}\right)$ with the magnitude of effect sizes determined as: small $\leq 0.01$, medium $\geq 0.06$, and large $\geq 0.14$ for $\eta^{2}$. Using a mixed model ANOVA, a sample size of 14 individuals per group was found to be sufficient to detect a moderate (Cohens $d=0.57$ ) group by time interaction effect of VO2peak with a level of significance at 0.05 and power of 0.8 .

\section{Results}

\section{Baseline characteristics}

Baseline characteristics for each group are presented in Table 1. No significant differences were found between the two groups at baseline, except of age $(p=0.005)$ with participants in CT-group being 15-year younger on average, HR-rest ( $p=0.032$ ) which was significantly higher in the CT-group and gender.

\section{Training characteristics}

No drop-outs and no any adverse events were recorded during the 3-time points of this study. Moreover, $100 \%$ adherence to the program was demonstrated for during of the 8-weeks. 
Table 1: Baseline characteristics of the two groups.

\begin{tabular}{|c|c|c|}
\hline Variable & Control-group $(n=15)$ & CT-group $(n=15)$ \\
\hline Gender & $M=10 ; F=5$ & $M=5 ; F=10$ \\
\hline Age (years) & $41.1(46.0)$ & $26.2(27.0)$ \\
\hline Height (cm) & $170.7 \pm 9.51$ & $167.3 \pm 11.15$ \\
\hline Weight (kg) & $87.0 \pm 17.43$ & $89.3 \pm 16.32$ \\
\hline BMI $\left(\mathbf{k g} / \mathrm{m}^{2}\right)$ & $29.7 \pm 5.44$ & $32.0 \pm 5.21$ \\
\hline BF\% & $37.2 \pm 10.39$ & $25.0 \pm 5.99$ \\
\hline BF (Kg) & $32.8 \pm 13.44$ & $38.8 \pm 12.05$ \\
\hline LBM\% $\%$ & $28.6 \pm 5.70$ & $25.0 \pm 5.99$ \\
\hline LBM (Kg) & $25.4(28.0)$ & $22.2(20.3)$ \\
\hline WC (cm) & $91.1 \pm 10.75$ & $93.1 \pm 9.94$ \\
\hline Hip Circumference (cm) & $105.3 \pm 9.53$ & $110.9 \pm 11.95$ \\
\hline WHR & $0.9 \pm 0.86$ & $0.8 \pm 0.78$ \\
\hline SBP $(\mathrm{mmHg})$ & $118.5(120.0)$ & $118.1(115.0)$ \\
\hline $\mathrm{DBP}(\mathrm{mmHg})$ & $80.7 \pm 11.94$ & $83.6 \pm 10.02$ \\
\hline MAP (mmHg) & $93.1 \pm 12.01$ & $93.5 \pm 7.15$ \\
\hline RPP (mmHg $\left.{ }^{*} b p m\right)$ & $8788.9 \pm 1707.54$ & $9881.9 \pm 1714.53$ \\
\hline HRrest (bpm) & $73.9 \pm 10.89$ & $83.2 \pm 11.65$ \\
\hline VO2peak (ml O $/ 2 / \mathrm{kg} / \mathrm{min})$ & $15.5 \pm 2.75$ & $15.6 \pm 1.82$ \\
\hline LDL (mg/dL) & $109.5 \pm 18.24$ & $113.3 \pm 6.54$ \\
\hline HDL (mg/dL) & $50.5 \pm 12.09$ & $46.6 \pm 9.06$ \\
\hline $\mathrm{TC}(\mathrm{mg} / \mathrm{dL})$ & $182.9(183.0)$ & $179.3(167.0)$ \\
\hline Glucose (mg/dL) & $95.0 \pm 9.81$ & $89.7 \pm 12.01$ \\
\hline Triglycerides (mg/dL) & $114.3 \pm 75.92$ & $95.9 \pm 37.19$ \\
\hline Right QUADS(N) & $175.1 \pm 42.48$ & $171.5 \pm 36.00$ \\
\hline Left QUADS(N) & $165.9 \pm 37.01$ & $178.3 \pm 50.04$ \\
\hline Right HAMS(N) & $162.7 \pm 34.69$ & $150.1 \pm 52.46$ \\
\hline Left HAMS(N) & $168.1 \pm 34.41$ & $162.9 \pm 33.51$ \\
\hline OMNI Scale & - & $8.7(0.82)$ \\
\hline
\end{tabular}

Data are mean \pm SD for normally distributed data or median (interquartile range) for non-normally distributed data.

Abbreviations: BMI: Body Mass Index; BF\%: Percentage of Body Fat; LBM\%: Percentage of Lean Body Mass; LBM-Kg: Lean Body Mass in kilograms; WC: Waist Circumference; WHR: Waist to Hip Ratio; SBP: Systolic Blood Pressure; DBP: Diastolic Blood Pressure; MAP: Mean Arterial Pressure; RPP: Rate Pressure Product; HRrest: Resting Heart Rate; VO ${ }_{2}$ peak: Maximal Oxygen Consumption; LDL: Low Density Lipoprotein; HDL: High Density Lipoprotein; TC: Total Cholesterol; QUADS: Quadriceps; HAMS: Hamstrings; N: Newton; mg/dl: Milligrams per decilitre; mmHg: Millimetre of Mercury; cm: Centimetre; F: Females; M: Males.

\section{Monitoring of physical activity}

At T-1 and T-2, 90\% ( $n=27)$ of participants were categorized at low-category and $10 \%(n=3)$ at moderatecategory of fitness level. Only one participant in controlgroup and 2 participants in CT-group had a moderate level of fitness. No changes in the physical activity of the participants occurred (outside of the training program in the CT-group).

\section{Body composition}

There was a significant group $\times$ time interaction in $\mathrm{BW}$ $\left(p=0.004, \eta^{2}=0.002\right)$, BMI $\left(p=0.008, \eta^{2}=0.002\right), B F \%(p$ $\left.=0.001, \eta^{2}=0.004\right), B F(K g)\left(p=0.004, \eta^{2}=0.004\right)$, LBM\% $\left(p=0.001, \eta^{2}=0.01\right)$ and Hip circumference $(p=0.012$, $\eta^{2}=0.001$ ). The $\%$ difference (Post-Pre value/Pre Value $\times 100 \%)$ in BW was $0.6 \%( \pm 1.01)$ for the control-group and $-2.5 \%( \pm 3.33)$ for the CT-group. Likewise, the \% differences for the BMI were $0.3 \%( \pm 1.81)$ and $-2.7 \%( \pm$ $3.19)$, for the BF\% were $3.7 \%( \pm 4.33)$ and $-3.7 \%( \pm 5.39)$, for the $\mathrm{BF}(\mathrm{Kg})$ were $4.3 \%( \pm 6.46)$ and $-5.9 \%( \pm 7.60)$, for the LBM\% were $-4.7 \%( \pm 5.82)$ and $3.3 \%( \pm 6.72)$, for the Hip were $0.4 \%( \pm 0.90)$ and $0.5 \%( \pm 0.92)$ for the controlgroup and the CT-group respectively. As evidence from the effect sizes these changes were small despite being highly significant. No group $\times$ time interaction was evident in LBM (kg), WC, and WHR Table 2.

\section{Cardiorespiratory fitness}

There was a significant group by time interaction for the $\mathrm{VO}_{2}$ peak $(p=0.001)$ with a small effect size $\eta^{2}=0.02$. CT-group improved by $11.1 \%$ ( \pm 8.45 ) in the 8-weeks of the intervention (T-1 to T-2), in contrast to $1.7 \%$ ( \pm 5.87) improvement in control-group. While, $\mathrm{VO}_{2}$ peak 
Table 2: Outcome variables before and after 8-weeks.

\begin{tabular}{|c|c|c|c|c|c|c|c|}
\hline & \multicolumn{2}{|c|}{ Control-group $(n=15)$} & \multicolumn{2}{|c|}{ CT-group $(n=15)$} & \multicolumn{3}{|l|}{ p-values } \\
\hline & Pre & Post & Pre & Post & Time & $\begin{array}{l}\text { Time } \\
\text { Group }\end{array}$ & Group \\
\hline Weight (kg) & $87.0 \pm 17.43$ & $87.4 \pm 17.14$ & $89.3 \pm 16.32$ & $87.2 \pm 16.46$ & $p=0.047$ & $p=0.004$ & $p=0.862$ \\
\hline BMI (kg/m²) & $29.7 \pm 5.44$ & $29.8 \pm 5.36$ & $32.0 \pm 5.21$ & $31.1 \pm 5.14$ & $p=0.018$ & $p=0.008$ & $p=0.364$ \\
\hline $\mathrm{BF} \%$ & $37.2 \pm 10.39$ & $38.5 \pm 10.59$ & $43.2 \pm 9.74$ & $41.8 \pm 10.18$ & $p=0.877$ & $p=0.001$ & $P=0.225$ \\
\hline BF-kg (kg) & $32.8 \pm 13.44$ & $34.0 \pm 13.53$ & $38.8 \pm 12.05$ & $36.7 \pm 11.85$ & $p=0.396$ & $p=0.004$ & $P=0.357$ \\
\hline LBM\% $\%$ & $28.6 \pm 5.70$ & $27.2 \pm 5.40$ & $25.0 \pm 5.99$ & $25.9 \pm 6.34$ & $p=0.373$ & $p=0.001$ & $p=0.252$ \\
\hline LBM-kg (kg) & $25.4(28.02)$ & $24.5(26.96)$ & $22.2(20.32)$ & $22.3(20.50)$ & $p=0221$ & - & $p=0.824$ \\
\hline WC (cm) & $91.1 \pm 10.75$ & $91.5 \pm 11.03$ & $93.1 \pm 9.94$ & $91.8 \pm 10.35$ & $p=0.351$ & $p=0.059$ & $p=0.762$ \\
\hline Hip (cm) & $105.3 \pm 9.53$ & $105.7 \pm 10.22$ & $110.9 \pm 11.95$ & $110.3 \pm 11.29$ & $p=0.740$ & $p=0.012$ & $p=0.212$ \\
\hline WHR & $0.9 \pm 0.86$ & $0.9 \pm 0.86$ & $0.8 \pm 0.78$ & $0.8 \pm 0.85$ & $p=0.249$ & $p=0.185$ & $p=0.286$ \\
\hline $\mathrm{SBP}(\mathrm{mmHg})$ & $118.5(120.0)$ & $121.5(123.0)$ & $118.1(115.0)$ & $116.6(110.0)$ & $P=0.144$ & & $p=0.002$ \\
\hline DBP (mmHg) & $80.7 \pm 11.94$ & $84.6 \pm 9.89$ & $83.6 \pm 10.02$ & $78.2 \pm 6.93$ & $p=0.661$ & $p=0.009$ & $p=0.592$ \\
\hline HR (bpm) & $73.9 \pm 10.89$ & $76.0 \pm 9.91$ & $83.2 \pm 11.65$ & $79.3 \pm 9.35$ & $p=0.312$ & $p=0.003$ & $p=0.103$ \\
\hline MAP (mmHg) & $93.1 \pm 12.01$ & $96.9 \pm 9.43$ & $93.5 \pm 7.15$ & $89.3 \pm 7.54$ & $p=0.796$ & $p=0.001$ & $p=0.282$ \\
\hline RPP (mmHg $\left.{ }^{*} b p m\right)$ & $\begin{array}{l}8788.9 \\
1707.54 \\
\end{array}$ & $\begin{array}{l}9173.2 \\
1279.28 \\
\end{array}$ & $\begin{array}{ll}9881.9 & \pm \\
1714.53 & \end{array}$ & $\begin{array}{ll}8863.9 & \pm \\
1468.54 & \end{array}$ & $p=0.026$ & $p=0.001$ & $p=0.483$ \\
\hline LDL (mg/dL) & $109.5 \pm 18.24$ & $116.5 \pm 30.74$ & $113.3 \pm 26.54$ & $108.5 \pm 18.80$ & $p=0.840$ & $p=0.267$ & $p=0.766$ \\
\hline HDL (mg/dL) & $50.5 \pm 12.09$ & $60.7 \pm 37.90$ & $46.6 \pm 9.06$ & $46.7 \pm 11.13$ & $p=0.249$ & $p=0.261$ & $p=0.169$ \\
\hline $\begin{array}{l}\text { Triglycerides } \\
\text { (mg/dL) }\end{array}$ & $114.3 \pm 75.92$ & $111.9 \pm 45.78$ & $95.9 \pm 37.19$ & $95.6 \pm 33.36$ & $p=0.866$ & $p=0.894$ & $p=0.314$ \\
\hline TC (mg/dL) & $182.9(21.94)$ & $183.9(48.09)$ & $179.3(29.45)$ & $174.3(23.79)$ & $p=0.853$ & - & $p=0.530$ \\
\hline Glucose (mg/dL) & $95.0 \pm 9.81$ & $100.9 \pm 0.40$ & $89.7 \pm 12.01$ & $96.8 \pm 8.69$ & $p=0.005$ & $p=0.781$ & $p=0.143$ \\
\hline LHAMS (N) & $168.1 \pm 34.41$ & $166.7 \pm 32.35$ & $162.9 \pm 33.51$ & $169.1 \pm 32.87$ & $p=0.013$ & $p=0.001$ & $p=0.913$ \\
\hline RHAMS (N) & $162.7 \pm 34.69$ & $164.2 \pm 36.26$ & $150.1 \pm 52.46$ & $166.2 \pm 32.18$ & $p=0.097$ & $p=0.161$ & $p=0.711$ \\
\hline LQUADS (N) & $165.9 \pm 37.01$ & $166.3 \pm 36.63$ & $178.3 \pm 50.04$ & $182.7 \pm 46.98$ & $p=0.013$ & $p=0.040$ & $p=0.367$ \\
\hline RQUADS (N) & $175.1 \pm 42.48$ & $176.6 \pm 42.79$ & $171.5 \pm 36.00$ & $175.1 \pm 34.41$ & $p=0.020$ & $p=0.327$ & $p=0.857$ \\
\hline $\begin{array}{l}\text { VO2peak }\left(\mathrm{ml} \mathrm{O}_{2} I\right. \\
\mathrm{kg} / \mathrm{min})\end{array}$ & $15.5 \pm 2.75$ & $15.8 \pm 2.83$ & $15.6 \pm 1.88$ & $17.3 \pm 2.44$ & $p=0.001$ & $p=0.001$ & $p=0.258$ \\
\hline
\end{tabular}

Data are means \pm SD for normally-distributed variables and median (interquartile range) for non-normally distributed variables; for non-normally distributed variables Time effect was assessed with Kruskal-Wallis Test and Group effect with Wilcoxon Test for each group. Only the $p$-value for CT group is presented in the table. Data in bold for the $p$ value indicate significant Group $\times$ Time interaction.

Abbreviations: BMI: Body Mass Index; BF\%: Percentage of Body Fat; BF-Kg: Body Fat in kilos; LBM\%: Percentage of Lean Body Mass; LBM-Kg: Lean Body Mass in kilos; WC: Waist circumference; cm: centimetres; WHR: Waist to hip ratio; SBP: Systolic Blood Pressure; DBP: Diastolic Blood Pressure; HR: Heart Rate; MAP: Mean Arterial Pressure; RPP: Rate Pressure Product; bpm: beats per minute; mmHg: millimetres of Mercury; LDL: Low Density Lipoprotein; HDL: High Density Lipoprotein; TC: Total Cholesterol; mg/dL: Milligrams per decilitre; N: Newton; HAMS: Hamstrings; QUADS: Quadriceps; $\mathrm{VO}_{2}$ peak: Maximal Oxygen Consumption.

decreased in both groups during the detraining period (T-2 to $\mathrm{T}-3)$ by $3.2 \%( \pm 4.41)$ and $2.6 \%( \pm 4.25)$ in the CT and control-group respectively it remained $7.5 \%$ ( \pm 8.86) higher than baseline in the CT-group. Pair wise comparisons showed only in the CT-group significant differences between T-1 to T-2 $(p=0.001)$ as well as T-2 to T-3 $(p=0.002)$ but not between T-1 to T-3 ( $p=$ 0.239) (Figure 1). Wilcoxon test demonstrated that the exertion (OMNI Scale) of participants in CT-group was significantly lower $(p=0.004)$ at the end of the intervention. Ten out of fifteen participants showed a percentage change in OMNI scale between $10 \%$ and $33 \%$ while five participants showed no change. The average
$\%$ improvement in exertion was $11.9 \%( \pm 10.85)$.

\section{Biochemical markers}

None of the biochemical markers showed any statistically significant group $\times$ time interaction $\left(\eta^{2}\right.$ from 0.001 to 0.016 ) (Table 2). The \% differences (Post-Pre value/PreValue $\times 100 \%)$ for the LDL were $7.5 \%( \pm 24.68)$ and $-0.4 \%( \pm 24.17)$, for the HDL were $17.5 \%( \pm 56.23)$ and $0.5 \%( \pm 15.71)$, for the TC were $1.6 \%$ ( \pm 24.99 ) and $-0.7 \%$ ( \pm 19.12$)$, for the triglycerides were $12.3 \%$ $( \pm 32.03)$ and $9.9 \%( \pm 47.84)$ and for the glucose were $6.5 \%( \pm 9.12)$ and $9.65 \%( \pm 17.35)$ for the control and CT-group respectively. 


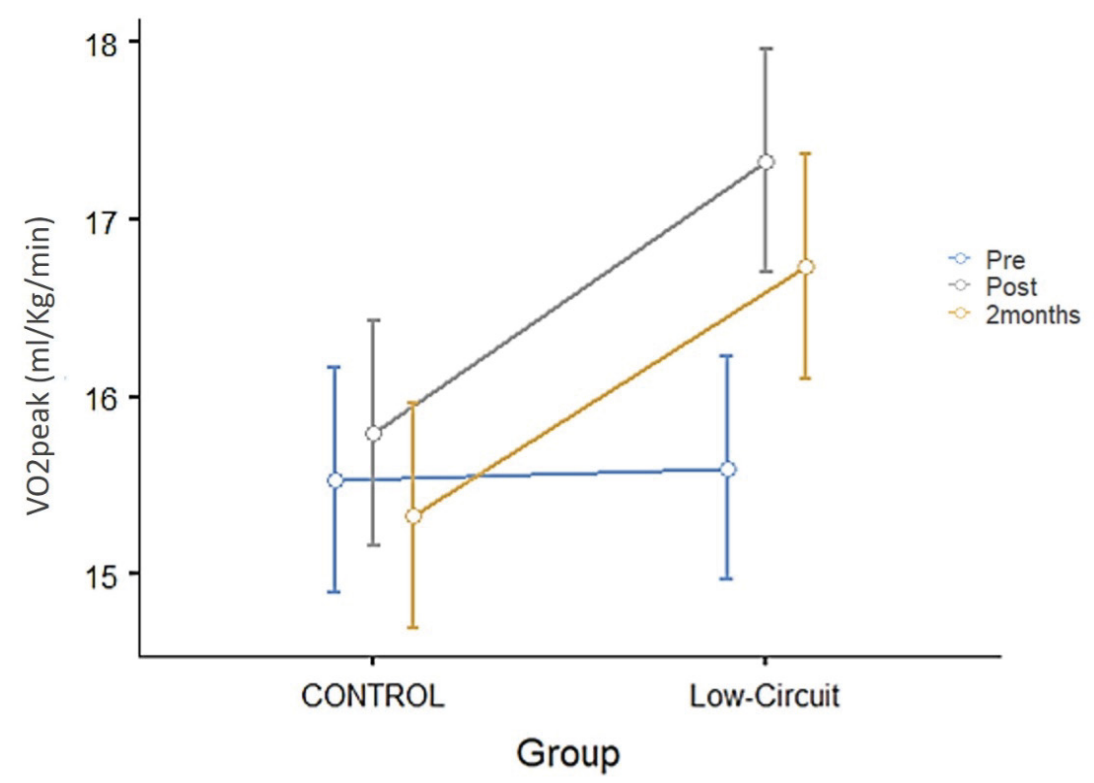

Figure 1: Changes in cardiorespiratory fitness (VO2peak) throughout the experimental period, from baseline (PRE) to 8-weeks post-intervention (POST) and at follow-up at 2-months (2M) for control-group and CT-group. Significant differences were found only in the Low Circuit group between T1 and T2 and T2 and T3 $(p<0.002)$. Values are in mean \pm SEM.S

\section{Physiological markers}

Significant group $\times$ time interactions were found for DBP $\left(p=0.009, \eta^{2}=0.06\right), \operatorname{HR}\left(p=0.003, \eta^{2}=0.02\right)$, $\operatorname{MAP}\left(p=0.001, \eta^{2}=0.05\right)$ and $\operatorname{RPP}\left(p=0.001, \eta^{2}=0.005\right)$ (Table 2). The \% difference (Post-Pre value/Pre Value $x$ $100 \%)$ in DBP was $5.6 \%( \pm 8.61)$ for the control-group and $-5.6 \%$ ( \pm 10.17$)$ for the CT-group. Likewise, the \% differences for the HR were $3.1 \%( \pm 4.6)$ and $-4.3 \%( \pm$ 6.50), for the MAP were $4.6 \%( \pm 7.23)$ and $-4.5 \%( \pm 4.25)$ and for the RPP were $6 \%( \pm 10.91)$ and $-10 \%( \pm 6.99)$ for the control-group and the CT-group respectively. SBP was not normally distributed and Kruskal-Wallis Test showed no changes over time. The control-group showed a $3 \%( \pm 5.98)(p=0.046)$ increase in SBP while CT-group had a $5.3 \%( \pm 5.46)(p=0.002)$ decrease in the 8-weeks of the exercise program (Table 2).

\section{Isometric strength}

Statistically significant group $\times$ time interactions were shown only at the non-dominant (Left) side ( $p=$ $0.001, \eta^{2}=0.003$ for the $L$ hamstrings and $p=0.040, \eta^{2}$ $=0.001$ for the $L$ quadriceps). No significant differences were found on the right side ( $\eta^{2}$ from 0.001 to 0.009 ). All these differences were small based on the classification of the effect size.

\section{Discussion}

This study examined whether CT can improve healthrelated parameters in apparently healthy overweight and obese individuals. The CT program with moderate intensity lasting for 8-weeks demonstrated no dropout rates, $100 \%$ adherence and zero adverse events.

\section{Cardiorespiratory fitness}

The increase in $\mathrm{VO}_{2}$ peak is considered as a strong prognostic marker of all-cause mortality and it is reported that an improvement in cardiorespiratory fitness by $1 \mathrm{ml} / \mathrm{kg} / \mathrm{min}$ is associated with a $9 \%$ reduction of cardiovascular mortality risk [10]. This study showed an average improvement of $1.7 \mathrm{ml} / \mathrm{kg} / \mathrm{min}$ (or $11 \%$ ) from $\mathrm{T}-1$ to $\mathrm{T}-2$ and $1.3 \mathrm{ml} / \mathrm{kg} / \mathrm{min}$ (or $7.5 \%$ ) T-1 to T-3 with only 16 sessions of 20-minutes of workout per session in eight weeks. Sperlich, et al. demonstrated that after 9-weeks of high intensity CT (2-6 series of functional exercises), 3 sessions per week led to improvements in $\mathrm{VO}_{2} \max (10.1 \%)$ of overweight women [41]. BallestaGarcia, et al. showed a difference of $3.4 \mathrm{ml} / \mathrm{kg} / \mathrm{min}$ in VO2max between the exercise group and the controlgroup with high intensity CT of $60 \mathrm{~min}$, performed twice a week for 18-weeks, in obese middle-aged women [56]. Batrakoulis, et al. reported that the VO2max of obese women improved from $26.1 \pm 4.4 \mathrm{ml}_{-} \mathrm{kg}^{-1}{ }_{-} \mathrm{min}$ at baseline to $33.1 \pm 4.8 \mathrm{ml}_{-} \mathrm{kg}^{-1} \mathrm{~min}^{-1}$ post-intervention following a CT program of higher intensity ( $\geq 65 \%$ of HRR) and of significantly higher volume (40-weeks) compared to the current study [57]. While the increases in $\mathrm{VO}_{2}$ max were higher in the previously mentioned studies, their protocols included longer and more intense sessions for longer period of time. Overall, the results of the current study suggest that the benefits of CT start to appear with significantly lower volume and intensity, suggesting that CT at moderate intensities $(50-60 \%$ of HRR) might be a time efficient alternative to HIIT or HICT with comparable health benefits and the potential of considerable increase in adherence. 


\section{Body composition}

The parameters of the exercise protocol were not sufficient to produce meaningful effects on the body composition variables. A series of meta-analysis $[58,59]$ reported that $\mathrm{CT}$ improves body composition in obese/ overweight population but the effect varied among the studies. Studies with comparable duration (8-weeks) showed significant improvement in BW, BMI and WHR in obese men $[43,60]$, but no effect on these body composition outcomes in obese women [44]. Moreover, body composition of obese/overweight men was improved after 4-weeks and 12-weeks of HICT (30-mins and 50 -min per session respectively) $[45,46]$. In addition, body composition improved after 8-weeks of moderate CT (60-min per session) in combination to green tea extract supplementation [61] in overweight middleaged men and after 12-weeks of moderate CT in obese women [62]. Other studies showed that the reduction of $\mathrm{BF}$ is greater in obese/overweight population when the exercise lasts for a longer period $[63,64]$ and a substantial weight loss ( $>5 \%$ reduction of $\mathrm{BW}$ ) requires an additional dietary intervention [64] or higher energy expenditure (at least 26 MET-hours). Collectively the results of this study, in the light of the previous literature, suggest that body composition might require higher volume and/ or intensity or moderate intensity with longer period or with additional nutritional intervention. Realistically though, high intensity exercise, for a long period of time and especially if it is combined with dietary restrictions is often difficult to maintain especially by obese/ overweight individuals [65]. An additional consideration that might explain the results of the current study on body composition might be the gender effect. Studies reported that males have a greater $\mathrm{BW}$ reduction than females when exercise is of similar duration and relative intensity $[66,67]$ possibly due to higher baseline values [68]. Most of the studies which showed positive effects of $\mathrm{CT}$ in body composition are in men while the current study included both men and women.

CT generally uses body weight resistance exercises and results in an optimal increase of LBM and a reduction of BF due to the increased muscular effort [69]. In this study the change in LBM\% was primarily mediated by the reduction of $\mathrm{BF}$ as the $\mathrm{LBM}(\mathrm{Kg})$ remained unchanged. This result is consistent with Kim, et al. Sadarzade, et al. and Lehnert, et al. who reported a non-significant difference between groups for this variable [62] despite the fact that resistance exercises have high energy demands [70]. The lack of effect in LBM is probably due to insufficient training volume (sets $\times$ reps $\times$ load), as a clear dose-response relationship exists between number of sets and increase in muscle mass [71].

\section{Physiological parameters}

Research has determined that perceived exertion is a suitable measure of intensity during and following both resistance and aerobic exercise. Participants in the current study showed an increased adaptation to the program as demonstrated by the reduction in OMNIscale. Although this was not amajor outcome of this study, the decreased rate of perceived exertion is likely to translate in improved functional ability and account for the high adherence and zero dropout rate of the program. Exertion is also influenced by psychological factors and it is strongly correlated with HR [72]. Exercise of sufficient time can affect the parasympathetic system and lead to an increase in stroke volume and a reduction in HR at rest [50]. HR and RPP are good predictors of myocardial oxygen consumption during exercise, reflecting the contractile state of the heart [73]. Findings of this study demonstrated a reduction of RPP in the CT-group compared to the control-group which was accompanied by corresponding changes in HR, SBP, DBP and MAP. Similar results in RPP, have been reported following CT for 12 -weeks (at $50-70 \%$ of HRR, 3x $40-65$ $\mathrm{min} /$ week) in obese women [74].

A positive effect in $\mathrm{BP}$ at rest can be expected following an aerobic exercise program, however the mechanism is different depending on the exercise variables. Nevertheless, there is no clear minimal dose of exercise for lowering BP or any clear independent effect of the key variables of exercise [64]. It is well established though that BP is decreased due to decreased activity of sympathetic nervous system and decreased peripheral resistance [75]. The observed $7 \mathrm{mmHg}$ decrease in SBP, is consistent with previously published data from a CT study involving obese and in normotensive populations [76]. DBP also improved post-intervention similar to another CT study lasting 12 -weeks. It should be highlighted that while these participants were overweight or obese this was a normotensive population and this improvement was not expected, since the physiological response of DBP to dynamic exercise is to maintain baseline levels, which were normal in this case. The decrease in SBP and DBP can potentially be due to a combination of central cardiac and peripheral adaptations. Interval training allows participants to challenge the pumping capability of the heart more than continuous exercise. MAP is a product of cardiac output and systemic vascular resistance. A possible explanation for the $4.5 \%$ improvement of MAP, is the lower resting HR post-intervention which can probably be attributed to a combination of an increased vagal tone [77] and a possible increase in plasma volume, leading to increase in stroke volume by the Frank-Starling mechanism $[78,79]$.

\section{Isometric strength}

The program used in this study utilised a series of several whole-body exercises and resulted in improved isometric muscle strength after only 20-minutes of exercise per session for 16 sessions only on the $L$ side. Since there was no change in LBM $(\mathrm{Kg})$ the improvement in strength was probably be due to neural adaptations. 
Previous studies showed that HIIT for 8-weeks and CT for 12-weeks increased back strength and grip strength in obese older women [80], middle-aged obese women [57] and female students [62]. Moreover, isometric strength improvements have been reported only in one limb following 12-weeks of CT in overweight women [81], similar to this study. As the majority of our participants were right side dominant $(86.67 \%)$ and dominant limbs are utilised more during the activities of daily living neural adaptations affected only the nondominant side [82]. A combination of higher volume or intensity is necessary for true and bilateral strength benefits with concomitant increase in muscle mass.

\section{Biochemical markers}

HDL and LDL lipoproteins are major indicators for the prediction of coronary artery disease [62]. CT-group demonstrated a reduction in LDL by $5 \mathrm{mg} / \mathrm{dL}$, which is consistent with the values reported in a series of metaanalyses by Kelley, et al. who found that routine exercise produced a clinically favourable but insignificant reduction in LDL by $4-6 \mathrm{mg} / \mathrm{dL}$ among various adult populations $[83,84]$. No effect was found in TC, probably due to the high variability of the measurements, which is unfortunate, as lowering TC by as little as $10 \%$, using a dietary or pharmacologic intervention was able to reduce cardiovascular disease incidence by $27 \%$ [85]. Other studies reported significant improvements in lipid profile of obese men and overweight/obese women following CT with progressive resistance (3x/week, 65$85 \%$ of 1 RM) (Kolahdouzi, et al.) or CT protocols with higher intensities and frequencies [86]. Moreover, studies using HICT of longer duration (12-weeks) and higher frequencies ( $3 \times /$ week) or weekly volume $(3 \times 30-$ min/week) reported improvements in lipid profile of middle-aged overweight [46] and obese men [45]. Although the role of nutrition in overweight and obese population is critical, two large meta-analyses found that HDL significantly improved by exercise even when confounding variables such as diet was considered $[87,88]$. The lack of significant effect of the current protocol in the biochemical variables might be because their values were normal at baseline which is supported by the results of a similar study by Kim, et al. in obese females. Alternatively, an CT program with higher duration and/or intensity is necessary to induce biochemical adaptations.

\section{Limitations}

The study limitations include the sample size which was small to allow a broader generalisation of the results. In addition, the participants were apparently healthy, so results might not be directly applicable to obese with risk factors or chronic diseases. Moreover, the study was probably underpowered to detect meaningful between group changes in secondary outcomes, as the power analysis was done based on the $\mathrm{VO}_{2}$ peak. Additionally, groups were not balanced in terms of age and gender, with a larger number of obese/overweight women in the CT-group therefore hormonal changes in both men and women [89] might have affected the results. Further, the study did not monitor daily nutrition (apart from the training sessions) and simply asked the participants to continue their normal diet, which might have influenced the results.

\section{Conclusions}

In summary, these findings suggest a safe, timeefficient exercise method and the lower end of training variables which are sufficient to promote health benefits in overweight/obese individuals without comorbidities or any other health-related risk factors. The results also suggest that 40-minutes of CT exercise per week, for 8-weeks can improve cardiorespiratory fitness. However additional training volume or more than 8-weeks are required for meaningful improvements in body composition, physiological and biochemical variables. Future studies should control for dietary intake and energy expenditure per day as they may be more important for body composition and lipid profile.

\section{References}

1. Collaborators GBDO, Afshin A, Forouzanfar $\mathrm{MH}$, Reitsma MB, Sur P, et al. (2017) Health Effects of Overweight and Obesity in 195 Countries over 25 Years. N Engl J Med 377: 13-27.

2. Chooi YC, Ding C, Magkos F (2019) The epidemiology of obesity. Metabolism 92: 6-10.

3. Guh DP, Zhang W, Bansback N, Amarsi Z, Birmingham $\mathrm{CL}$, et al. (2009) The incidence of co-morbidities related to obesity and overweight: A systematic review and metaanalysis. BMC Public Health 9: 88.

4. Jakicic JM, Rogers RJ, Davis KK, Collins KA (2018) Role of Physical Activity and Exercise in Treating Patients with Overweight and Obesity. Clin Chem 64: 99-107.

5. Johns DJ, Hartmann-Boyce J, Jebb SA, Aveyard $P$, Behavioural Weight Management Review Group (2014) Diet or exercise interventions vs combined behavioral weight management programs: A systematic review and meta-analysis of direct comparisons. J Acad Nutr Diet 114: 1557-1568.

6. Avenell A, Brown TJ, McGee MA, Campbell MK, Grant AM, et al. (2004) What interventions should we add to weight reducing diets in adults with obesity? A systematic review of randomized controlled trials of adding drug therapy, exercise, behaviour therapy or combinations of these interventions. J Hum Nutr Diet 17: 293-316.

7. Chaput JP, Klingenberg L, Rosenkilde M, Gilbert JA, Tremblay A, et al. (2011) Physical activity plays an important role in body weight regulation. J Obes 2011: 360257.

8. Yang YJ (2019) An Overview of Current Physical Activity Recommendations in Primary Care. Korean J Fam Med 40: 135-142.

9. Andersen RE, Jakicic JM (2009) Interpreting the physical activity guidelines for health and weight management. $J$ Phys Act Health 6: 651-656.

10. Reljic D, Frenk F, Herrmann HJ, Neurath MF, Zopf Y (2021) Effects of very low volume high intensity versus moderate 
intensity interval training in obese metabolic syndrome patients: A randomized controlled study. Sci Rep 11: 2836

11. Whyte LJ, Gill JM, Cathcart AJ (2010) Effect of 2 weeks of sprint interval training on health-related outcomes in sedentary overweight/obese men. Metabolism 59: 14211428.

12. Trilk JL, Singhal A, Bigelman KA, Cureton KJ (2011) Effect of sprint interval training on circulatory function during exercise in sedentary, overweight/obese women. Eur J Appl Physiol 111: 1591-1597.

13. Whyte LJ, Ferguson C, Wilson J, Scott RA, Gill JM (2013) Effects of single bout of very high-intensity exercise on metabolic health biomarkers in overweight/obese sedentary men. Metabolism 62: 212-219.

14. Hood MS, Little JP, Tarnopolsky MA, Myslik F, Gibala MJ (2011) Low-volume interval training improves muscle oxidative capacity in sedentary adults. Med Sci Sports Exerc 43: 1849-1856.

15. Gillen JB, Percival ME, Ludzki A, Tarnopolsky MA, Gibala MJ (2013) Interval training in the fed or fasted state improves body composition and muscle oxidative capacity in overweight women. Obesity (Silver Spring 21: 2249-2255.

16. Fisher G, Brown AW, Bohan Brown MM, Alcorn A, Noles C, et al. (2015) High Intensity Interval- vs Moderate Intensity-Training for Improving Cardiometabolic Health in Overweight or Obese Males: A Randomized Controlled Trial. PLoS One 10: e0138853.

17. Russomando L, Bono V, Mancini A, Terracciano A, Cozzolino F, et al. (2020) The Effects of Short-Term High-Intensity Interval Training and Moderate Intensity Continuous Training on Body Fat Percentage, Abdominal Circumference, BMI and VO2max in Overweight Subjects. J Funct Morphol Kinesiol 5: 41

18. Shiraev T, Barclay G (2012) Evidence based exercise clinical benefits of high intensity interval training. Aust Fam Physician 41: 960-962.

19. Perri MG, Anton SD, Durning PE, Ketterson TU, Sydeman SJ, et al. (2002) Adherence to exercise prescriptions: Effects of prescribing moderate versus higher levels of intensity and frequency. Health Psychol 21: 452-458.

20. Farias-Junior LF, Browne RAV, Freire YA, Oliveira-Dantas FF, Lemos T, et al. (2019) Psychological responses, muscle damage, inflammation, and delayed onset muscle soreness to high-intensity interval and moderate-intensity continuous exercise in overweight men. Physiol Behav 199: 200-209.

21. Batacan RB, Duncan MJ, Dalbo VJ, Tucker PS, Fenning AS (2017) Effects of high-intensity interval training on cardiometabolic health: a systematic review and meta-analysis of intervention studies. Br J Sports Med 51: 494-503.

22. Hannan AL, Hing W, Simas V, Climstein M, Coombes JS, et al. (2018) High-intensity interval training versus moderateintensity continuous training within cardiac rehabilitation: a systematic review and meta-analysis. Open Access $\mathrm{J}$ Sports Med 9: 1-17.

23. Vella CA, Taylor K, Drummer D (2017) High-intensity interval and moderate-intensity continuous training elicit similar enjoyment and adherence levels in overweight and obese adults. Eur J Sport Sci 17: 1203-1211.

24. Wewege M, van den Berg R, Ward RE, Keech A (2017) The effects of high-intensity interval training vs. moderateintensity continuous training on body composition in overweight and obese adults: A systematic review and meta-analysis. Obes Rev 18: 635-646.
25. Andreato LV, Esteves JV, Coimbra DR, Moraes AJP, de Carvalho $T$ (2019) The influence of high-intensity interval training on anthropometric variables of adults with overweight or obesity: A systematic review and network meta-analysis. Obes Rev 20: 142-155.

26. Bull FC, Al-Ansari SS, Biddle S, Borodulin K, Buman MP, et al. (2020) World Health Organization 2020 guidelines on physical activity and sedentary behaviour. Br J Sports Med 54: 1451-1462.

27. Heisz JJ, Tejada MG, Paolucci EM, Muir C (2016) Enjoyment for High-Intensity Interval Exercise Increases during the First Six Weeks of Training: Implications for Promoting Exercise Adherence in Sedentary Adults. PLoS One 11: e0168534.

28. Hughes DC, Ellefsen S, Baar K (2018) Adaptations to Endurance and Strength Training. Cold Spring Harbor Perspectives in Medicine 8: 029769.

29. Anek A, Kanungsukasem V, Bunyaratavej N (2015) Effects of Aerobic Step Combined with Resistance Training on Biochemical Bone Markers, Health-Related Physical Fitness and Balance in Working Women. J Med Assoc Thai 98: 42-51.

30. Ribeiro AS, Tomeleri CM, Souza MF, Pina F, Schoenfeld BJ, et al. (2015) Effect of resistance training on C-reactive protein, blood glucose and lipid profile in older women with differing levels of RT experience. Age (Dordr) 37: 109.

31. Takenami E, Iwamoto S, Shiraishi N, Kato A, Watanabe $Y$, et al. (2019) Effects of low-intensity resistance training on muscular function and glycemic control in older adults with type 2 diabetes. J Diabetes Investig 10: 331-338.

32. Tomeleri CM, Souza MF, Burini RC, Cavaglieri CR, Ribeiro AS, et al. (2018) Resistance training reduces metabolic syndrome and inflammatory markers in older women: A randomized controlled trial. J Diabetes 10: 328-337.

33. Willis LH, Slentz CA, Bateman LA, Shields AT, Piner LW, et al. (2012) Effects of aerobic and/or resistance training on body mass and fat mass in overweight or obese adults. $J$ Appl Physiol 113: 1831-1837.

34. Orange ST, Madden LA, Vince RV (2020) Resistance training leads to large improvements in strength and moderate improvements in physical function in adults who are overweight or obese: A systematic review. Journal of Physiotherapy 66: 214-224.

35. Slentz CA, Bateman LA, Willis LH, Shields AT, Tanner CJ, et al. (2011) Effects of aerobic vs. resistance training on visceral and liver fat stores, liver enzymes, and insulin resistance by HOMA in overweight adults from STRRIDE AT/RT. Am J Physiol Endocrinol Metab 301: 1033-1039.

36. Feito Y, Heinrich KM, Butcher SJ, Poston WSCJS (2018) High-intensity functional training (HIFT): definition and research implications for improved fitness. Sports 6: 76.

37. Alcaraz PE, Sanchez-Lorente J, Blazevich AJ (2008) Physical performance and cardiovascular responses to an acute bout of heavy resistance circuit training versus traditional strength training. J Strength Cond Res 22: 667671.

38. Marcos-Pardo PJ, Orquin-Castrillon FJ, Gea-Garcia GM, Menayo-Antunez R, Gonzalez-Galvez N, et al. (2019) Effects of a moderate-to-high intensity resistance circuit training on fat mass, functional capacity, muscular strength, and quality of life in elderly. A randomized controlled trial. Sci Rep 9: 7830.

39. Batrakoulis A, Jamurtas AZ, Georgakouli K, Draganidis D, 
Deli CK, et al. (2018) High intensity, circuit-type integrated neuromuscular training alters energy balance and reduces body mass and fat in obese women: A 10-month trainingdetraining randomized controlled trial. PLoS One 13: e0202390.

40. Bocalini DS, Lima LS, de Andrade S, Madureira A, Rica RL, et al. (2012) Effects of circuit-based exercise programs on the body composition of elderly obese women. Clin Interv Aging 7: 551-556.

41. Sperlich B, Wallmann-Sperlich B, Zinner C, Von Stauffenberg V, Losert H, et al. (2017) Functional HighIntensity Circuit Training Improves Body Composition, Peak Oxygen Uptake, Strength, and Alters Certain Dimensions of Quality of Life in Overweight Women. Front Physiol 8: 172.

42. Paoli A, Pacelli F, Bargossi AM, Marcolin G, Guzzinati S, et al. (2010) Effects of three distinct protocols of fitness training on body composition, strength and blood lactate. J Sports Med Phys Fitness 50: 43-51.

43. Kolahdouzi S, Baghadam M, Kani-Golzar FA, Saeidi A, Jabbour G, et al. (2019) Progressive circuit resistance training improves inflammatory biomarkers and insulin resistance in obese men. Physiol Behav 205: 15-21.

44. Franklin NC, Robinson AT, Bian JT, Ali MM, Norkeviciute E, et al. (2015) Circuit resistance training attenuates acute exertion-induced reductions in arterial function but not inflammation in obese women. Metab Syndr Relat Disord 13: $227-234$

45. Miller MB, Pearcey GE, Cahill F, McCarthy H, Stratton SB, et al. (2014) The effect of a short-term high-intensity circuit training program on work capacity, body composition, and blood profiles in sedentary obese men: a pilot study. Biomed Res Int 2014: 191797.

46. Paoli A, Pacelli QF, Moro T, Marcolin G, Neri M, et al. (2013) Effects of high-intensity circuit training, low-intensity circuit training and endurance training on blood pressure and lipoproteins in middle-aged overweight men. Lipids Health Dis 12: 131

47. Wilke J, Kaiser S, Niederer D, Kalo K, Engeroff T, et al. (2019) Effects of high-intensity functional circuit training on motor function and sport motivation in healthy, inactive adults. Scand J Med Sci Sports 29: 144-153.

48. Hecksteden A, Faude O, Meyer T, Donath L (2018) How to Construct, Conduct and Analyze an Exercise Training Study?. Front Physiol 9: 1007.

49. Tanaka H, Monahan KD, Seals DR (2001) Age-predicted maximal heart rate revisited. J Am Coll Cardiol 37: 153-156.

50. Thompson PD, Arena R, Riebe D, Pescatello LS (2013) ACSM's new preparticipation health screening recommendations from ACSM's guidelines for exercise testing and prescription, ninth edition. Curr Sports Med Rep 12: $215-217$

51. Bautista IJ, Chirosa IJ, Tamayo IM, Gonzalez A, Robinson JE, et al. (2014) Predicting Power Output of Upper Body using the OMNI-RES Scale. J Hum Kinet 44: 161-169.

52. Burns SP, Breuninger A, Kaplan C, Marin H (2005) Handheld dynamometry in persons with tetraplegia: comparison of make- versus break-testing techniques. Am J Phys Med Rehabil 84: 22-29.

53. Ray CA, Rea RF, Clary MP, Mark AL (1993) Muscle sympathetic nerve responses to dynamic one-legged exercise: effect of body posture. Am J Physiol 264: 1-7.

54. Sagiv M, Ben-Sira D, Sagiv A, Werber G, Rotstein A (1994)
Left ventricular responses during prolonged treadmill walking with heavy load carriage. Med Sci Sports Exerc 26: 285-288.

55. Arya A, Maleki M, Noohi F, Kassaian E, Roshanali F (2005) Myocardial oxygen consumption index in patients with coronary artery disease. Asian Cardiovasc Thorac Ann 13: 34-37.

56. Ballesta-Garcia I, Martinez-Gonzalez-Moro I, RamosCampo DJ, Carrasco-Poyatos M (2020) High-Intensity Interval Circuit Training Versus Moderate-Intensity Continuous Training on Cardiorespiratory Fitness in MiddleAged and Older Women: A Randomized Controlled Trial. Int J Environ Res Public Health 17: 1805.

57. Ballesta-Garcia I, Martinez-Gonzalez-Moro I, Rubio-Arias JA, Carrasco-Poyatos M (2019) High-Intensity Interval Circuit Training Versus Moderate-Intensity Continuous Training on Functional Ability and Body Mass Index in Middle-Aged and Older Women: A Randomized Controlled Trial. Int J Environ Res Public Health 16.

58. Schwingshackl L, Dias S, Strasser B, Hoffmann G (2013) Impact of different training modalities on anthropometric and metabolic characteristics in overweight/obese subjects: a systematic review and network meta-analysis. PLoS One 8: 82853 .

59. Seo YG, Noh HM, Kim SY (2019) Weight loss effects of circuit training interventions: A systematic review and metaanalysis. Obes Rev 20: 1642-1650.

60. Safarzade A, Alizadeh H, Bastani Z (2020) The effects of circuit resistance training on plasma progranulin level, insulin resistance and body composition in obese men. Horm Mol Biol Clin Investig 41.

61. Bagheri R, Rashidlamir A, Ashtary-Larky D, Wong A, Grubbs $B$, et al. (2020) Effects of green tea extract supplementation and endurance training on irisin, pro-inflammatory cytokines, and adiponectin concentrations in overweight middle-aged men. Eur J Appl Physiol 120: 915-923.

62. Kim J, Ko Y, Seo T, Kim Y (2018) Effect of circuit training on body composition, physical fitness, and metabolic syndrome risk factors in obese female college students. $J$ Exerc Rehabil 14: 460-465.

63. Cornelissen VA, Smart NA (2013) Exercise training for blood pressure: a systematic review and meta-analysis. $J$ Am Heart Assoc 2: e004473.

64. Wasfy MM, Baggish AL (2016) Exercise Dose in Clinical Practice. Circulation 133: 2297-2313.

65. Lean MEJ, Astrup A, Roberts SB (2018) Making progress on the global crisis of obesity and weight management. BMJ 361: 2538.

66. Donnelly JE, Hill JO, Jacobsen DJ, Potteiger J, Sullivan DK, et al. (2003) Effects of a 16-month randomized controlled exercise trial on body weight and composition in young, overweight men and women: The Midwest Exercise Trial'. Arch Intern Med 163: 1343-1350.

67. Potteiger JA, Jacobsen DJ, Donnelly JE, Hill JO (2003) Glucose and insulin responses following 16 months of exercise training in overweight adults: the Midwest Exercise Trial. Metabolism 52: 1175-1181.

68. Williams RL, Wood LG, Collins CE, Callister R (2015) Effectiveness of weight loss interventions--is there a difference between men and women: a systematic review. Obes Rev 16: 171-186.

69. Tremblay A, Simoneau JA, Bouchard C (1994) Impact of 
exercise intensity on body fatness and skeletal muscle metabolism. Metabolism-Clinical and Experimental 43: 814818.

70. Reis VM, Junior RS, Zajac A, Oliveira, DR (2011) Energy cost of resistance exercises: an uptade. J Hum Kinet 29: 33-39.

71. Schoenfeld BJ, Grgic J, Ogborn D, Krieger JW (2017) Strength and Hypertrophy Adaptations Between Low- vs. High-Load Resistance Training: A Systematic Review and Meta-analysis. J Strength Cond Res 31: 3508-3523.

72. Chen YL, Chen CC, Hsia PY, Lin SK (2013) Relationships of Borg's RPE 6-20 scale and heart rate in dynamic and static exercises among a sample of young Taiwanese men. Percept Mot Skills 117: 971-982.

73. Lamina S, Okoye GC, Ezema Cl, Anele TI, Ezugwu AU (2013) Effect of interval training program on rate-pressure product in the management of hypertension in black African male subjects: A randomized controlled trial. Nigerian Journal of Basic and Clinical Sciences 10: 17.

74. Kang HJ, Lee YS, Park DS, Kang DH (2012) Effects of 12 week circuit weight training and aerobic exercise on body composition, physical fitness, and pulse wave velocity in obese collegiate women. Soft Computing 16: 403-410.

75. Cavalcante MA, Bombig MTN, Filho B, de Camargo Carvalho AC, de Paola AA, et al. (2007) Quality of life of hypertensive patients treated at an outpatient clinic. Arquivos brasileiros de cardiologia 89: 245.

76. Pescatello LS, MacDonald HV, Lamberti L, Johnson BT (2015) Exercise for Hypertension: A Prescription Update Integrating Existing Recommendations with Emerging Research. Curr Hypertens Rep 17: 87.

77. Blomqvist CG, Saltin B (1983) Cardiovascular adaptations to physical training. Annual Review of Physiology 45: 169189.

78. Convertino V, Brock P, Keil L, Bernauer E, Greenleaf J (1980) Exercise training-induced hypervolemia: role of plasma albumin, renin, and vasopressin. J Appl Physiol Respir Environ Exerc Physiol 48: 665-669.

79. Goodman JM, Liu PP, Green HJ (2005) Left ventricular adaptations following short-term endurance training. J Appl Physiol 98: 454-460.
80. Smith-Ryan AE, Trexler ET, Wingfield HL, Blue MN (2016) Effects of high-intensity interval training on cardiometabolic risk factors in overweight/obese women. J Sports Sci 34: 2038-2046

81. Lehnert M, Stastny P, Sigmund M, Xaverova Z, Hubnerova $B$, et al. (2015) The effect of combined machine and body weight circuit training for women on muscle strength and body composition. J Phys Educ Sport 15: 561.

82. Balshaw TG, Massey GJ, Maden-Wilkinson TM, MoralesArtacho AJ, McKeown A, et al. (2017) Changes in agonist neural drive, hypertrophy and pre-training strength all contribute to the individual strength gains after resistance training. Eur J Appl Physiol 117: 631-640.

83. Kelley GA, Kelley KS, Tran ZVJJowsh (2004) Aerobic exercise and lipids and lipoproteins in women: a metaanalysis of randomized controlled trials. J Womens Health 13: $1148-1164$

84. Kelley GA, Kelley KS (2009) Impact of progressive resistance training on lipids and lipoproteins in adults: $A$ meta-analysis of randomized controlled trials. Prev Med 48: 9-19.

85. Law MR, Wald NJ, Thompson SJB (1994) By how much and how quickly does reduction in serum cholesterol concentration lower risk of ischaemic heart disease?. BMJ 308: 367-372.

86. Fett CA, Fett WC, Marchini JS (2009) Circuit weight training vs jogging in metabolic risk factors of overweight/obese women. Arq Bras Cardiol 93: 519-525.

87. Durstine JL, Grandjean PW, Davis PG, Ferguson MA, Alderson NL, et al. (2001) Blood lipid and lipoprotein adaptations to exercise: a quantitative analysis. Sports Med 31: 1033-1062.

88. Leon AS, Sanchez OA (2001) Response of blood lipids to exercise training alone or combined with dietary intervention. Med Sci Sports Exerc 33: 528-509.

89. Stevens J, Katz E, Huxley R (2010) Associations between gender, age and waist circumference. Eur $\mathrm{J}$ Clin Nutr 64: 6-15. 